\title{
Editorial
}

\section{Steroid Use, Abuse and Withdrawal Protocol}

Steroids are hormones have got natural (endogenous) and synthetic (exogenous) sources. Source of natural steroids are adrenal cortex, testes and ovaries. Synthetic anabolic steroids are the substances that are similar in nature to male sex hormones. Adrenal cortex hormones, Glucocorticoid, Mineral corticoids and androgen are the natural steroid. ${ }^{1,2}$ Steroids are used in clinical practice in various purposes, such as replacement therapy, in the treatment of autoimmune disorders, treatment and management of inflammatory disease, management of allergic reaction other medical uses of sex steroids include helps with delayed puberty, impotence, building lean muscle mass, anemia, osteoporosis, improve weight loss due to illness, among others. Steroids (Glucocorticoid) are life saving hormone. ${ }^{2,3,4,5}$ The act of using steroid in dishonest way or for the wrong purpose is called misuse. Abuse is the synonym of misuse. In our perspective steroids are misused by various ways. Traditional healers (Kabiraj) misuse steroids by making puria (mixtures of Glucocorticoid and other bronchodilators) for the management of asthma. They use it as a magical drug for all diseases. Some street hawkers and pharmacy worker sell steroids to the young people for gaining weight and look healthy. Steroids also use for increasing appetite.

Anabolic androgenic steroids are prone to abuse, because of their bodybuilding properties. The misuse and abuse of this drug is prevalent, especially in sports, and predominately in bodybuilding and weightlifting. ${ }^{1,2}$ Athletes have been known to abuse steroids to enhance performance, build muscle and mass, and improve physical appearance. However, because of its dangerous side effects, many professional sports have now banned steroid use and instituted mandatory drug testing, specifically to test for steroid use in professional athletes.

Steroids, similar to testosterone in the body, can cause many health related problems, including cancer, high blood pressure, irregular heartbeat, and even death. ${ }^{2,6,7,8,9}$ Other negative effects from steroid use are that the user is prone to bouts of aggression and uncontrolled anger, mood swings, paranoia, and severe irritability. Another common trend with steroid use is that people who regularly abuse steroids may seek other medications or drugs in order to alleviate some of the side effects of using the steroids. Because of this, steroids can easily be a gateway drug to more hard-core drug abuse.
Even when taken under a doctor's care, and in small does, steroids can cause irreversible damage to the user's body, even if the user stops taking steroids. This is one of the reasons steroids only have limited therapeutic and medical purposes. Steroids can be toxic, especially when abused or taken in large doses, and can cause permanent damage to the liver and kidneys. ${ }^{11}$ Additionally, steroids have been known to cause seizures, coronary artery disease, and other heart related problems. Heart complications when using steroids are increased with excessive physical exertion, making it even more dangerous for athletes to abuse steroids.

Another common myth about steroids is that just taking steroids alone will increase muscle mass and make the user 'buff' and built, but nothing could be further from the truth. Even when taking steroids, the user must perform muscle building exercises in order to see any appreciable results besides weight gain. This is something many teens do not understand, believing that simply taking steroids will change the shape of their bodies. This is simply not true. Even with steroid use, hard work and body building exercises and weight lifting are still required to achieve the physique desired. Steroid use alone will not cause this.

While steroid use might help enhance physical appearance, body building exercises, when properly performed, will produce the same results without the dangerous side effects. Steroids are not necessary for bodybuilding. Exercise and determination go much further and are much safer than steroids. Steroids are not cheap either. The can be taken orally or intramuscularly through injections. Oil based steroid injections can remain in the body for as long as 6 months, but the effects of the steroids may only last for a few days. Oral steroids only last for a few hours. The cost per dosage is high, and for the steroid injections may cost as much as \$100-500.00 per dose, and many people take more than one dose per day.

Many men may think that taking steroids will make them more virile, but in actually, steroid use can cause genital atrophy, genital swelling, sexual dysfunction, sterility, impotence, prostate enlargement. Very sexy stuff there, right?Bottom line is, the only way to build healthy muscle and mass is to properly exercise and eat a balanced, nutritious diet. Steroids may provide a temporary fix to problems of physique, but death is a price too high to pay to

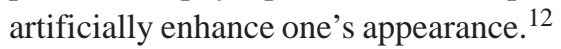




\section{Suggested Plan For Steroid Replacement In Patients Withdrawing From Chronic Corticosteroid Therapy ${ }^{13}$

\author{
Duration of Glucocorticoid Treatment \\ $<3$
} \\ wk}

$\begin{array}{ll}\text { Dose }(\mathbf{m g} & <\mathbf{3} \\ \text { pred/day) } & \text { wk(*) } \\ <7.5 \mathrm{mg} & \text { Can } \\ & \text { stop }\end{array}$

$5-7.5 \mathrm{mg}$

$<5 \mathrm{mg}$
Reduce rapidly e.g., $3.5 \mathrm{mg}$ every $3-4$ days

THEN

Reduce by $1 \mathrm{mg}$ every $2-4$ wk

THEN

Reduce by $1 \mathrm{mg}$ every 2-4 wk
OR Convert $5 \mathrm{mg}$ pred to $\mathrm{HC} 20 \mathrm{mg}$ and $<3$ wk $\downarrow$ by $2.5 \mathrm{mg} / \mathrm{kg}$ to $10 \mathrm{mg}$ for $2-3 \mathrm{mo}$

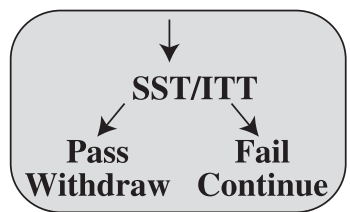

* Beware frequent steroid courses, e.g., in asthma pred, Prednisolone; SST, short synacthen test, ITT, insulin tolerance test

The protocol of steroid withdrawal: If the duration of steroid is $<3$ wks we can stop the steroid in any time. If the duration is $>3 \mathrm{wks}$ and dose of steroid is $>7.5 \mathrm{mg} /$ day to reduce the dose $2.5 \mathrm{mg} / 3-4$ days until the dose of steroid reaches below $7.5 \mathrm{mg} /$ day. Then we have to reduce the dose $1 \mathrm{mg}$ of steroid every 24 wks or we can convert $5 \mathrm{mg}$ prednisolone into $20 \mathrm{mg}$ hydrocortisone and then reduce the dose of hydrocortisone $2.5 \mathrm{mg}$ every week until reduced to $10 \mathrm{mg} /$ day. Then after every 2-3 months short synecthin test (SST) or Insulin tolerance test should be done. If the patient pass, that is (Glucocorticoid $>550 \mathrm{mg}$ ) then withdraw the steroid completely. It not or fail to pass. Then the dose of steroid should remain as before and then again after $>2-3$ months, Short SynecthinTest and Insulin Tolerance Test should be done again. ${ }^{13}$

\section{Conclusion:}

Misuse and abuse of steroid is a public health problem. Longterm use of steroid in supraphysiological dose may cause permanent atrophy of the adrenalgland.The steroid use is very important and sometimes life-saving. But on the other hand when it is misused or abused it can cause injury to health and demoralization of society. It is the massage that, steroid use needs awareness regarding its good effect and side effect which can minimize the steroid use,reduce the misuse and abuse.

\section{MAMannan}

Professor \& Head, Department of Endocrinology, Dhaka Medical College Hospital, Dhaka.

\section{References:}

1. Pickup ME:Clinical pharmacokinetics of Prednisolone and Prednisone,Clin Pharmacokinetic 4:111-128,1979.

2. Axelrod L. Glucocorticoid therapy,Medicine (Baltimore) 1976; 55: 39-65.
3. Dioid Paolo M,AgozzinoM,ToniC,etal.Sudden anabolic steroid abuse-related death

4. Kokkevi A,Fotiou A,Chileva A,et al. Daily exercise and anabolic steroids use in adolescents:a cross -national European study.Subst use Misuse.Aug27 2008;1

5. Gambertogilo JG,Amend WJCJr,BanetLZ: Pharmacokinetic and bioavailability ofprednisolone and prednisone in healthy volunteer and patient:a review , J pharmacokinetic biopharm 8:1-52,1980.

6. Lewis GP,JuskoWJ,Burke CW, et al: prednisolone side effect and serum protein level :collaborative study,Lancet 2:778781,1971

7. Fineschi V,Riezzo I,Centini F,et al. Sudden cardiac death during anabolic steroid abuse: Morphologic and toxicologic findings in two fatal cases of bodybuilders.Int J Legal Med. Jan 2007;121(1):48-53.

8. Lau DH,Stiles MK,John B et al. Atrial fibrillation and anabolic steroid abuse.Int J Cardiol.Apr 25 2007;117(2):86-7.

9. Achar S. Rostamian A,Narayan SM,Cardiac and metabolic effects of anabolic-androgenic steroid abuse on lipids,blood pressure,left ventricular dimension, and rhythm.Am J Cardiol Sep 15 2010:106(6):893-901.

10. Baggish AL, Weiner RB, KanayamaG,HudsonJI, Picard MH, Hutter AM Jr,et al Long term anabolic -androgenic steroid use is associated with left ventricular dysfunction. Circ Heart Fail,Jul 1 2010;3(4):472-6.

11. Schwingel PA,Cotrim HP,Salles BR,Almeida CE,Nachef B,et al. Anabolic androgenic steroid: a possible new risk factor of toxicant associated fatty liver disease. Liver Int. Mar 2011; 31(3): 348-53.

12. Http://Voices.Yahoo.Com/Steroid-Abuse-Good-Bad-UglySteroid-92929.Html. [Accessed on 09.11.2013].

13. KrokenbergH.M,Melmed S, Polonsky K.S, Larsen P.R, 2008; Williams Textbook of Endocrinology, $11^{\text {th }}$ edition, Canada, Saunders. The adrenalcortex: Paul M. Stewart, Suggested plan for steroid replacement in patients withdrawing from chronic corticosteroid therapy. 